\title{
BMJ Open Experiences of telehealth e-mentoring within postgraduate musculoskeletal physical therapy education in the UK and Canada: a protocol for parallel mixed-methods studies and cross-cultural comparison
}

Nicola R Heneghan (D , ${ }^{1}$ Madeleine Nazareth, ${ }^{1}$ Wendy J Johnson, ${ }^{2,3}$ Isaak Tyros, ${ }^{1}$ Jackie Sadi, ${ }^{4}$ Heather Gillis, ${ }^{4}$ Alison B Rushton (i) ${ }^{4}$

To cite: Heneghan NR, Nazareth M, Johnson WJ, et al. Experiences of telehealth $\mathrm{e}$ mentoring within postgraduate musculoskeletal physical therapy education in the UK and Canada: a protocol for parallel mixed-methods studies and cross-cultural comparison. BMJ Open 2021;11:e042602. doi:10.1136/

bmjopen-2020-042602

- Prepublication history and additional material for this paper are available online. To view these files, please visit the journal online (http://dx.doi. org/10.1136/bmjopen-2020042602).

Received 13 July 2020

Revised 19 January 2021

Accepted 25 January 2021

Check for updates

(C) Author(s) (or their employer(s)) 2021. Re-use permitted under CC BY-NC. No commercial re-use. See rights and permissions. Published by BMJ.

For numbered affiliations see end of article.

Correspondence to

Dr Nicola R Heneghan;

n.heneghan@bham.ac.uk

\section{ABSTRACT}

Introduction Mentored clinical practice is central to demonstrating achievement of International Educational Standards in advanced musculoskeletal physical therapy. While traditionally delivered face-to-face, telehealth ementoring is a novel alternative to offering this unique pedagogy to facilitate mentee critical reflection, deeper learning and enhanced knowledge translation to optimise patient care. With COVID-19 resulting in widespread adoption of telehealth and access to mentors often limited by geography or cost, the potential value of telehealth e-mentoring needs investigating. To investigate the experiences and outcomes of multiple stakeholders (student mentees, mentors and patients) engaged in musculoskeletal physical therapy telehealth e-mentoring across two universities (UK and Canada).

Methods and analysis Using case study design, we will use sequential mixed methods involving qualitative and quantitative components based on existing evidence. To examine the influence of telehealth e-mentoring on health outcomes in patients with musculoskeletal complaints, we will use patient-reported outcomes for satisfaction, patient empowerment and change in musculoskeletal health. We will conduct semistructured interviews to explore the development of critical thinking, clinical reasoning, communication skills and confidence of students engaged in telehealth e-mentoring. To explore the mentor acceptability and appropriateness of telehealth e-mentoring, we will conduct a focus group in each site. Finally, we will include a focus group of participants from each site to allow a crosscultural comparison of findings to inform international stakeholders. Quantitative data will be analysed using descriptive statistics (median and IQR) to describe changes in outcome data and qualitative data will be analysed following the Framework Method.

Ethics and dissemination This study has ethical approval from both institutions: the University of Birmingham (ERN_20-0695) and Western University (2020-116233-47832). Findings will be published in a peer-reviewed journal and disseminated to key
Strengths and limitations of this study

- This is the first study to explore the experiences of multiple stakeholders engaged in telehealth ementoring for postgraduate physical therapy.

- The mixed-methods case study design enables detailed exploration of experiences and outcomes involving all stakeholders as participants.

- Cross-cultural comparison is enabled through the use of multiple study site settings across countries/ continents.

- Findings will be specific to physical therapy, the two universities and their respective countries, thus limiting transferability to other professions, settings and countries.

stakeholders in musculoskeletal physical therapy education and practice.

\section{INTRODUCTION}

Telehealth, which encompasses 'telemedicine', 'telecare', 'telerehabilitation' 'teleconsult' and 'e-health', ' delivers healthcare using virtual technology and online communication platforms and can move beyond traditional practice settings, ${ }^{2}{ }^{3}$ to overcome barriers to accessing healthcare such as time, geography and costs of specialist services. ${ }^{14}$ Considerable evidence exists to support its effectiveness (clinical and cost) and acceptability for improving health outcomes for patients, including reducing hospital admissions. ${ }^{135}$ Patient satisfaction with telehealth in muscoloskeletal (MSK) physical therapy is widely reported as high ${ }^{3}$ and in fact recent trials reported higher levels than face-to-face care. ${ }^{36}$ Yet, widespread adoption of telehealth within physical therapy has been slow ${ }^{3}$ and to the 
author's knowledge has not yet been used as a medium to support postgraduate professional development in MSK physical therapy. While a high level of psychomotor skills are deemed a core construct of MSK advanced practice, ${ }^{7}$ a considerable number of other core constructs (e.g. high levels of clinical reasoning, background knowledge and self-analysis as well as patient-centred approach, critical approach to practice etc.) could be achieved via telehealth. ${ }^{6-8}$ With evidence of good concurrent validity between telehealth-based physiotherapy assessment and that involving face-to-face assessment for a range of outcomes (pain, swelling, joint mobility, muscle strength etc.) ${ }^{9}$ and a range of studies within medicine supporting that $75 \%-83 \%$ of diagnoses are derived from the patient history data alone, ${ }^{10}{ }^{11}$ learning outcomes could arguably be achieved through telehealth e-mentoring.

In MSK physical therapy, the International Federation of Orthopaedic Manipulative Physical Therapists (IFOMPT) sets the International Educational Standards $^{12}$; being then operationalised through approved programmes in the UK by the Musculoskeletal Association of Chartered Physiotherapists (MACP) and in Canada by the Canadian Association of Manipulative Physical Therapy (CAMPT). Mentored clinical practice (MCP) is central to demonstrating achievement of these educational standards. Using a framework of clinical reasoning, students as mentees, facilitated by a mentor, are able to integrate new skills and knowledge (procedural and propositional) acquired within a university setting into their assessment and management of patients with MSK complaints in a practice setting. MCP offers a unique pedagogy to facilitate critical reflection, deeper learning and enhanced knowledge translation ${ }^{13}$ to optimise patient care. Improvements in patient outcomes for those physical therapists who had completed fellowships with a component of $\mathrm{MCP}^{14}$ and clinical trial data supporting its clinical effectiveness now exist. ${ }^{15}$

MCP is highly dependent on specialist input and suitably qualified MSK physical therapy mentors. Access to these mentors has become increasingly difficult in the UK with many of these highly trained mentors often working at an advanced practice level or in consultant practitioner roles; therefore being involved in managing or leading large physical therapy services with no capacity for mentorship. Additionally, in Canada the geography of the country makes access to mentors challenging and limits opportunities for mentees to access mentors based on location. This often results in professional isolation for physical therapists practising in rural areas. ${ }^{13}$ Likewise, students report increasing difficulties when negotiating time away from work (costs, access, impact on service etc.) to complete the MCP component of IFOMPTapproved programmes. The COVID-19 pandemic has further compounded these issues forcing us to rethink how we approach healthcare delivery and mentorship. This has resulted in a shift of all initial contact MSK physical therapy being delivered as telehealth via remote digital consultation (telephone consultation and/or video consultation) and a stepped rationale for escalation to face-to-face consultation. ${ }^{3}$ In light of these changes, alternative MCP approaches are urgently required to fulfil student and IFOMPT-approved programmes' needs, including the use of telehealth e-mentoring.

While a myriad of approaches are used (face-to-face, e-mentoring and telehealth), telehealth e-mentoring could be an adjunct to conventional face-to-face MCP approaches beyond COVID-19, to better prepare students through novel approaches to delivery of specialist MSK physical therapy; using remote and digital consultation as part of clinical triage in outpatient healthcare consultations. ${ }^{16-18}$

Where telehealth e-mentoring has been introduced in light of COVID-19, we urgently need to evaluate its acceptability, appropriateness and satisfaction from the stakeholder perspectives of patients, mentors and students. This potentially offers a novel approach for professional growth and development, integrating knowledge and skills acquired in a university setting into clinical practice.

\section{Aim}

To investigate the experiences of postgraduate Masters (MSc) physical therapy students, their mentors and patients with MSK complaints engaged telehealth e-mentoring (consultation/rehabilitation) in a UK and Canadian university setting.

\section{Objectives}

1. To examine the influence of telehealth e-mentoring on health outcomes in patients with MSK complaints.

2. To explore the development of critical thinking, clinical reasoning, communication skills and confidence of postgraduate students engaged in telehealth ementoring.

3 . To explore the mentor acceptability and appropriateness of telehealth e-mentoring to facilitate student development towards achievement of IFOMPT Educational Standards.

4. To conduct a cross-cultural comparison of findings to inform international stakeholders.

\section{METHODS AND ANALYSIS \\ Design and methods}

Using case study design, ${ }^{19}$ we will use sequential mixed qualitative and quantitative methods to investigate telehealth e-mentoring as part of postgraduate physiotherapy education (figure 1). Data collection will take place from May 2020 through to December 2021. Details of the telehealth e-mentoring are detailed in figure 2. In the absence of reporting guidelines for mixed-methods studies, the study is designed in line with the COnsolidated criteria for REporting Qualitative research. ${ }^{20}$ and STrengthening the Reporting of Observational Studies in Epidemiology statement ${ }^{21}$ for qualitative and observational study designs, respectively.

Semistructured interviews will be used to explore development of critical thinking, clinical reasoning, 


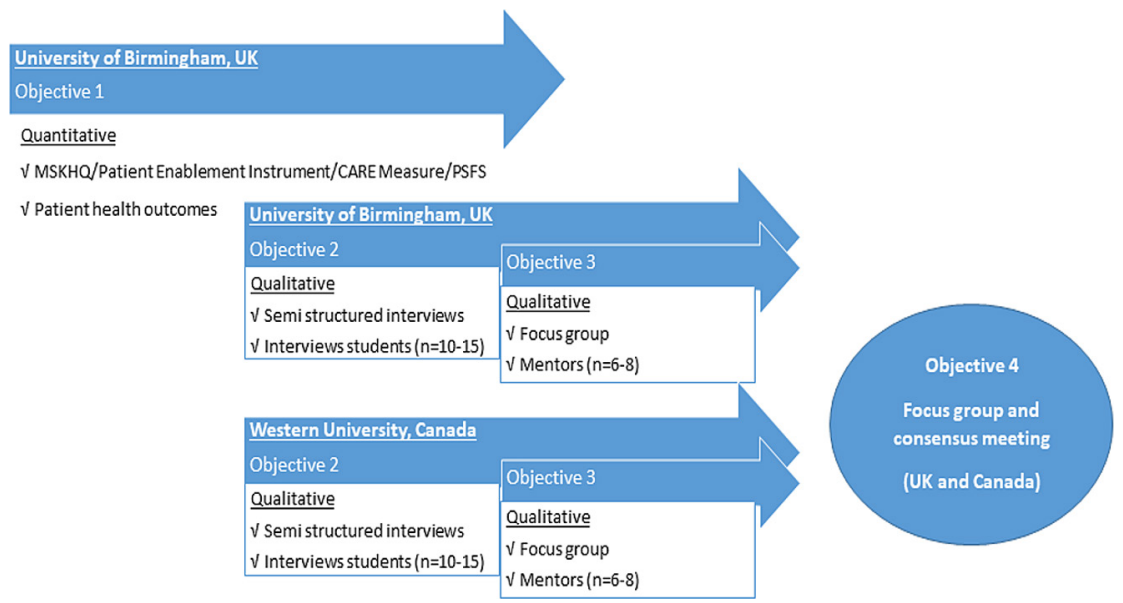

Figure 1 Exploratory sequential case study design. CARE, Consultation and Relational Empathy; MSK-HQ, Musculoskeletal Health Questionnaire; PSFS, Patient Specific Functional Scale.

communication skills and confidence of postgraduate students, patient-reported outcomes to explore patient experiences and changes in MSK health following telehealth e-mentoring, a focus groups to explore mentor acceptability and appropriateness of telehealth e-mentoring and a focus group for cross-cultural comparison of all data.

\section{Participant recruitment and eligibility for each site}

All participants must be able to communicate fluently in English, with access to a video consultation platform and able and willing to give informed consent.

- Patients (expected $n=\sim 50+$ ) who self-refer to the UK University Advanced Manipulative Physical Therapy telehealth service with a MSK complaint will be invited to participate.

- Students $(\mathrm{n}=10-15)$ from the cohort of postgraduate students registered on the respective
IFOMPT-approved programmes who are registered on the MCP module/course will be invited to participate in a semistructured interview. Purposive sampling will be used to ensure a variance in the profile of participants with respect to age, gender, geographical and clinical experience on entry to the programme.

- Clinical mentors from the University of Birmingham and Western University programme $(n=6-8)$ will be invited to participate in the focus group.

Exclusion criteria for patient participants include those who are not reporting an MSK complaint, for example, stroke rehabilitation. Mentees and mentors without licence to practise and professional indemnity insurance for the respective countries will be excluded.

\section{Study setting}

Two sites will be used to collect data: the University of Birmingham, UK and Western University, London,

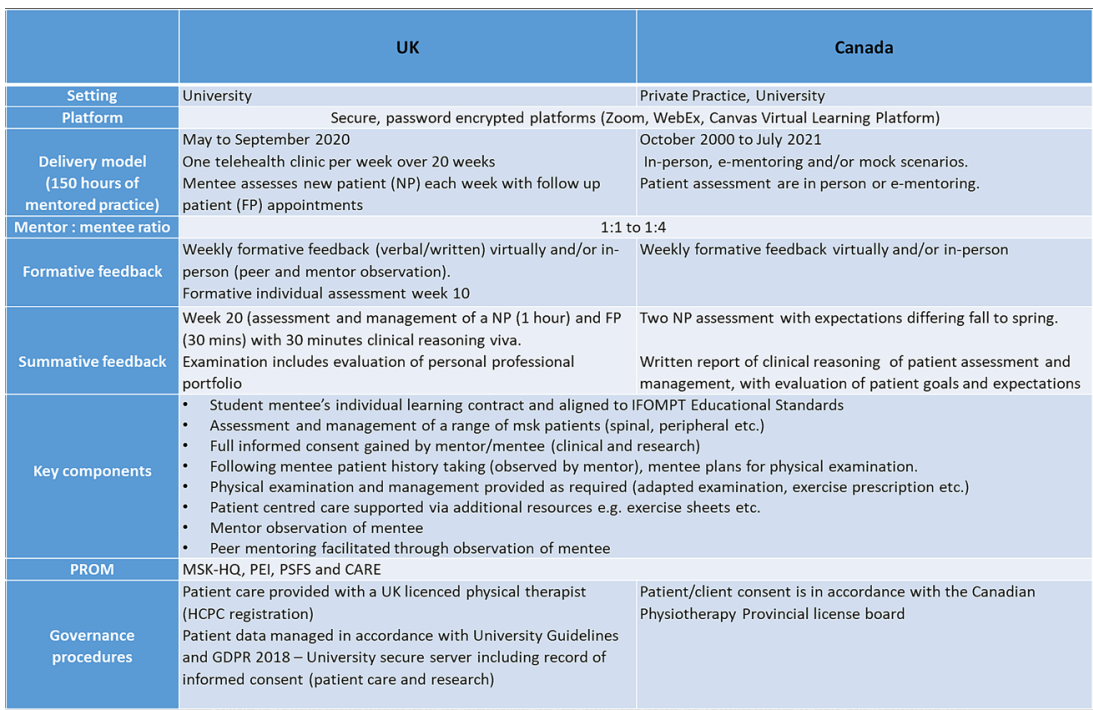

Figure 2 Description of telehealth. CARE, Consultation and Relational Empathy; GDPR, General Data Protection Regulation; HCPC, Health and Care Professions Council; IFOMPT, International Federation of Orthopaedic Manipulative Physical Therapists; MSK-HQ, Musculoskeletal Health Questionnaire; PEI, Patient Enablement Instrument; PROM, patient-reported outcome measure; PSFS, Patient Specific Functional Scale. 
Ontario, Canada. Both universities host programmes that offer eligibility to IFOMPT via nationally accredited programmes in MSK physical therapy: Birmingham since 2004 and Western since 2007. Each site experiences unique challenges to fulfilment of MCP thereby affording a different lens on the potential merits of telehealth e-mentoring. A video consulting platform e.g. Zoom (password protected) will be used to conduct interviews and the focus group.

\section{Data collection and procedures}

Objective 1: longitudinal observational study (UK only)

Participants (patients) will be invited to participate in the study and complete the following questionnaires on initial assessment and/or discharge or onward referral (online supplemental file 1). Outcomes were selected to enable characterisation of the patient population and evaluation of their experiences of telehealth (self-care ability and satisfaction).

1. Patient health: the 15-item Musculoskeletal Health Questionnaire $^{22}$ is a recently developed patientreported outcome measure for use with patients with MSK conditions and Patient Specific Functional Scale ${ }^{23}$ is a patient-specific valid, reliable and responsive outcome measure for patients with MSK complaints (participants selecting two activities to rate; 2-item) ${ }^{23} 24$

2. Patient enablement: the 6-item Patient Enablement Instrument ${ }^{25}$ is a well-established measure of selfcare ability in first contact and primary care consultations. ${ }^{25} 26$ It has also been reported to demonstrate fair content validity, construct validity and internal consistency in patients with chronic MSK symptoms. ${ }^{27}$

3. Patient-reported experience measures (10-item): rating of patient satisfaction will include key telehealth patient satisfaction themes, namely overall satisfaction, audiovisual quality and the Consultation and Relational Empathy measure. $^{28}$

\section{Objective 2: semistructured interviews}

Participants (students) will be invited to participate in a semistructured interview which will last $45-60 \mathrm{~min}$. The interview will explore students' expectations and perceptions prior, during and after the period of telehealth e-mentoring, as well as exploring their individual experiences and beliefs around their development of clinical reasoning. The topic guide (online supplemental file 2) was informed by existing evidence including the M-Level health education Logic Model ${ }^{29}$ and the core constructs of MSc level practice in MSK physical therapy. ${ }^{7}$

\section{Objective 3: focus group}

Participants (mentors) will be invited to participate in a focus group which will last $60-90 \mathrm{~min}$. The focus group will explore acceptability and appropriateness of telehealth e-mentorship to facilitate student development towards achievement of IFOMPT Educational Standards. The topic guide will be developed inductively from the interim analysis of semistructured interview data.

\section{Objective 4: focus group}

Researchers and representatives from participant groups (patient, mentor and mentee) will be invited to participate in a focus group which will last 90-120 min. The focus group will explore key themes and outcomes from country-specific findings as part of a cross-cultural analysis. The topic guide for this will be developed inductively from the analysis of data from focus group, semistructured interviews and patient outcomes in each country.

\section{Data management and data analysis}

Quantitative data, including participant demographics, will be analysed descriptively using means and SD to determine change on pre-management and post-management, and to characterise the patient population who presented for telehealth in this study. Interviews and focus group will be audio recorded and transcribed verbatim. Posttranscription, response clarification will be completed to enhance accuracy and trustworthiness of participant views (member checking), with field notes used to maintain contextual details and non-verbal responses during data analysis/interpretation. ${ }^{20}$

Qualitative data will be analysed using the wellestablished Framework Method..$^{30}$ This is a seven-stage process for qualitative data management and analysis involving (1) transcription, (2) familiarisation with the interview, (3) coding, (4) developing a working analytical framework, (5) applying the analytical framework, (6) charting data into the framework matrix and (7) interpreting the data. This approach will allow us to examine and understand the experiences of telehealth and e-mentoring from interview and focus group data. The method highlights the importance of involvement of individuals with experience in qualitative research, therefore experienced qualitative researchers will be involved at every stage of analysis.

\section{Data storage, access and disposal}

All quantitative data from the study will be collected using a bespoke online questionnaire, incorporating the measures as detailed above. Audio data will be transferred securely, transcribed by an approved service. Participant data will be stored confidentially for 10 years on password-protected computers that can only be accessed by the researchers, and in accordance with General Data Protection Regulation, the Data Protection Act 2018 and University of Birmingham's research governance frameworks in the UK and Western University Health Science Research Ethics Board.

\section{Patient and public involvement}

While conceived during the COVID-19 pandemic as a means of supporting ongoing student progression and development, the study is informed through many years of working (clinically and educationally) with patients, postgraduate students and MACP/CAMPT mentors. Given the novelty of this approach to postgraduate education, our prestudy consultation involved students, 
patients, practitioners and representatives from relevant professional, ethical and legal bodies.

Mentors, mentees and patient representatives from each country will be invited to participate in the focus group to support cross-cultural analysis and interpretation of results, including key recommendations. Key stakeholders in postgraduate education for MSK physical therapists may also be contacted for their contribution and insight to help aid analysis and interpretation of results including the MACP and CAMPT. Patient and public involvement in the full study will be reported using the GRIPP2-SF when disseminating study results. ${ }^{31}$

\section{Ethics and dissemination}

Ethical approval has been granted by the respective ethics committees at the University of Birmingham (15/05/2020 ref ERN_20-0695) for the UK and at Western University, London, Canada (21/12/2020 ref 2020-116233-47832). All participants will sign a consent form and receive a participant information sheet prior to participation. They will have the right to withdraw from the study at any point and up to 4 weeks after data collection is completed. Patient participants will be advised that any involvement will not impact on any current and future healthcare. There are minimal risks associated with this study. When presenting the study findings, pseudonyms will be used to protect the participants' identities. Any protocol deviations will be documented. The findings from this research will be disseminated to key stakeholders in postgraduate MSK physical therapy education nationally and internationally, including MACP and IFOMPT.

\section{DISCUSSION}

This protocol outlines the rationale and methodology of a mixed-methods case study design across two countries to explore the experiences of telehealth e-mentoring within postgraduate MSK physical therapy education. The lead researchers for each site have considerable experience of postgraduate education in MSK physical therapy and experiences of the proposed research methodologies including relevant publications. ${ }^{7} 1229$

Findings from this international study will place a spotlight on the MACP and CAMPT as leading and collaborating internationally in innovative approaches to enable fulfilment of IFOMPT Educational Standards. The crosscultural analysis will allow us to consider and share best practice experiences in telehealth e-mentoring, recognising that telehealth is well established in Canada as a means of delivering healthcare. We plan to explore how this and other cultural factors may influence the experiences of stakeholders to inform recommendations to IFOMPT for the adoption of telehealth e-mentoring in other member countries. Findings will inform the advancement of curriculum design in advanced MSK physical therapy postgraduate education, specifically overcoming the known limitations of existing approaches to MCP and to support development of advanced clinical reasoning using an authentic alternative to conventional approaches. It is anticipated that the findings from this study will also improve the access to mentors with specialist skills and knowledge globally, thus improving the educational fulfilment of student mentees and in turn patient outcomes.

\section{Limitations}

Where data collection is occurring across two sites (country and setting) at different time points, the experiences for all participants may differ across sites. Likewise those leading the interviews and focus groups across sites will differ. Steps will be taken to minimise the influence of these factors on the cross-cultural analysis, including use of a co-written standardised topic guide, collaboration through stages of data analysis and interpretation. Furthermore, this will be specifically explored with participants from both sites involved in the final focus group.

\section{Author affiliations}

${ }^{1}$ Centre of Precision Rehabilitation for Spinal Pain (CPR Spine), School of Sport, Exercise and Rehabilitation Sciences, University of Birmingham, Birmingham, UK

${ }^{2}$ Faculty of Health Sciences and Sport, University of Stirling, Stirling, UK ${ }^{3}$ Edinburgh Community Physiotherapy Service, NHS Lothian, Edinburgh, UK ${ }^{4}$ School of Physical Therapy, Western University, London, Ontario, Canada

Twitter Nicola R Heneghan @HeneghanNicola and Alison B Rushton @abrushton

Contributors NRH is the $\mathrm{Cl}$ leading the protocol development, analyses and dissemination. NRH and ABR are lead researchers for each site, ensuring rigour and quality in project management. NRH, WJ, MN, IT, JS, HG and ABR have contributed to the design and development of the protocol and have contributed to the manuscript draft. All authors have read, provided feedback and approved the final manuscript.

Funding The authors have not declared a specific grant for this research from any funding agency in the public, commercial or not-for-profit sectors.

Competing interests None declared.

Patient and public involvement statement The study is informed through many years of working (clinically and educationally) with patients, postgraduate students and MACP/CAMPT mentors. Given the novelty of this approach to postgraduate education, our pre-study consultation involved, students, patients, practitioners, and representatives from relevant professional, ethical and legal bodies.

Patient consent for publication Not required.

Ethics approval University of Birmingham Ethics Committee for the UK (15/05/2020 ref ERN_20-0695) and Health Science Research Ethics Board, Western University, London, Canada (21/12/2020 ref 2020-116233-47832)

Provenance and peer review Not commissioned; externally peer reviewed.

Supplemental material This content has been supplied by the author(s). It has not been vetted by BMJ Publishing Group Limited (BMJ) and may not have been peer-reviewed. Any opinions or recommendations discussed are solely those of the author(s) and are not endorsed by BMJ. BMJ disclaims all liability and responsibility arising from any reliance placed on the content. Where the content includes any translated material, BMJ does not warrant the accuracy and reliability of the translations (including but not limited to local regulations, clinical guidelines, terminology, drug names and drug dosages), and is not responsible for any error and/or omissions arising from translation and adaptation or otherwise.

Open access This is an open access article distributed in accordance with the Creative Commons Attribution Non Commercial (CC BY-NC 4.0) license, which permits others to distribute, remix, adapt, build upon this work non-commercially, and license their derivative works on different terms, provided the original work is properly cited, appropriate credit is given, any changes made indicated, and the use is non-commercial. See: http://creativecommons.org/licenses/by-nc/4.0/. 


\section{ORCID iDs}

Nicola R Heneghan http://orcid.org/0000-0001-7599-3674

Alison B Rushton http://orcid.org/0000-0001-8114-7669

\section{REFERENCES}

1 Tenforde AS, Hefner JE, Kodish-Wachs JE, et al. Telehealth in physical medicine and rehabilitation: a narrative review. $\mathrm{Pm} R$ 2017:9:S51-8

2 Chartered Society of Physiotherapy. What is telehealth? 2020 Available: https://www.csp.org.uk/professional-clinical/digitalphysiotherapy/telehealth

3 Cottrell MA, Russell TG, OLeary SP. Telehealth for musculoskeletal physiotherapy. Musculoskelet Sci Pract 2020;48:102193.

4 Dario AB, Moreti Cabral A, Almeida L, et al. Effectiveness of telehealth-based interventions in the management of non-specific low back pain: a systematic review with meta-analysis. Spine J 2017;17:1342-51.

5 Holland A. Telehealth reduces hospital admission rates in patients with COPD. J Physiother 2013;59:129.

6 Cottrell MA, Galea OA, O'Leary SP, et al. Real-Time telerehabilitation for the treatment of musculoskeletal conditions is effective and comparable to standard practice: a systematic review and metaanalysis. Clin Rehabil 2017;31:625-38.

7 Rushton A, Lindsay G. Defining the construct of masters level clinical practice in manipulative physiotherapy. Man Ther 2010;15:93-9.

8 Cottrell MA, Hill AJ, O'Leary SP, et al. Service provider perceptions of telerehabilitation as an additional service delivery option within an Australian neurosurgical and orthopaedic physiotherapy screening clinic: a qualitative study. Musculoskelet Sci Pract 2017;32:7-16.

9 Mani S, Sharma S, Omar B, et al. Validity and reliability of Internetbased physiotherapy assessment for musculoskeletal disorders: a systematic review. J Telemed Telecare 2017;23:379-91.

10 Ohm F, Vogel D, Sehner S, et al. Details acquired from medical history and patients' experience of empathy--two sides of the same coin. BMC Med Educ 2013;13:67.

11 Keifenheim KE, Teufel M, Ip J, et al. Teaching history taking to medical students: a systematic review. BMC Med Educ 2015;15:159.

12 International Federation of orthopaedic manipulative physical therapists. IFOMPT standards document, 2016. Available: https:// www.ifompt.org/STANDARD+COMPLIANCE++TRAINING.html

13 Ezzat AM, Maly MR. Building passion develops meaningful mentoring relationships among Canadian physiotherapists. Physiother Can 2012;64:77-85.

14 Rodeghero J, Wang Y-C, Flynn T, et al. The impact of physica therapy residency or fellowship education on clinical outcomes for patients with musculoskeletal conditions. J Orthop Sports Phys Ther 2015;45:86-96

15 Williams A, Rushton A, Lewis JJ, et al. Evaluation of the clinical effectiveness of a work-based mentoring programme to develop clinical Reasoning on patient outcome: a stepped wedge cluster randomised controlled trial. PLoS One 2019;14:e0220110.

16 Greenhalgh T, Wherton J, Shaw S, et al. Video consultations for covid-19. BMJ 2020;368:m998.

17 Thornton J. Covid-19: how coronavirus will change the face of general practice forever. BMJ 2020;368:m1279.

18 Nitkunan A, Paviour D, Nitkunan T. COVID-19: switching to remote neurology outpatient consultations. Pract Neurol 2020;20:222-4.

19 Miles AH. Qualitative data analysis: an expanded sourcebook. Beverley Hills: Sage, 1994.

20 Tong A, Sainsbury P, Craig J. Consolidated criteria for reporting qualitative research (COREQ): a 32-item checklist for interviews and focus groups. Int J Qual Health Care 2007;19:349-57.

21 von Elm E, Altman DG, Egger M, et al. Strengthening the reporting of observational studies in epidemiology (STROBE) statement: guidelines for reporting observational studies. BMJ 2007;335:806-8.

22 Hill JC, Kang S, Benedetto E, et al. Development and initial cohort validation of the arthritis research UK musculoskeletal health questionnaire (MSK-HQ) for use across musculoskeletal care pathways. BMJ Open 2016;6:e012331.

23 Horn KK, Jennings S, Richardson G, Van Vliet D, et al. The patientspecific functional scale: psychometrics, clinimetrics, and application as a clinical outcome measure. J Orthop Sports Phys Ther 2012;42:30-D17.

24 Nicholas P, Hefford C, Tumilty S. The use of the patient-specific functional scale to measure rehabilitative progress in a physiotherapy setting. J Man Manip Ther 2012;20:147-52.

25 Howie JG, Heaney DJ, Maxwell M, et al. A comparison of a patient enablement instrument (PEI) against two established satisfaction scales as an outcome measure of primary care consultations. Fam Pract 1998;15:165-71.

26 McKinstry B, Colthart I, Walker J. Can doctors predict patients' satisfaction and enablement? A cross-sectional observational study. Fam Pract 2006;23:240-5.

27 Enthoven P PA, Ludvigsson ML, Wibault J. Validity, internal consistency and self-rated change of the patient enablement instrument in patients with chronic musculoskeletal pain. J Rehabil Med 2019;51:587-97.

28 Mercer SW, Maxwell M, Heaney D, et al. The consultation and relational empathy (care) measure: development and preliminary validation and reliability of an empathy-based consultation process measure. Fam Pract 2004;21:699-705.

29 Madi M, Hamzeh H, Griffiths M, et al. Exploring taught masters education for healthcare practitioners: a systematic review of literature. BMC Med Educ 2019;19:340.

30 Gale NK, Heath G, Cameron E, et al. Using the framework method for the analysis of qualitative data in multi-disciplinary health research. BMC Med Res Methodol 2013;13:117.

31 Staniszewska S, Brett J, Simera I, et al. GRIPP2 reporting checklists: tools to improve reporting of patient and public involvement in research. BMJ 2017;358:j3453. 